
A. V. Gushchin ${ }^{1}$, O. S. Kalistratova ${ }^{1}$, A. I. Maleeva ${ }^{1}$, V. A. Kuropatov ${ }^{2}$, D. N. Yemelyanov ${ }^{1}$ ${ }^{1}$ Lobachevsky State University of Nizhny Novgorod, 23 Gagarin Av., Nizhny Novgorod, 603950, Russia,
${ }^{2}$ G. A. Razuvaev Institute of Organometallic Chemistry RAS, 49 Tropinina St., Nizhny Novgorod, 603137, Russia
E-mail: gushchin4@yandex.ru

\title{
Tert-butyl-diphenylmethylnitroxyl radical formation with the reaction of triphenylbismuth dicrotonate and $\mathrm{C}$-phenyl-N-tert-butylnitrone in benzene
}

$$
\mathrm{Ph}_{3} \mathrm{Bi}(\mathrm{V}) \stackrel{\text { hv }}{\longrightarrow}[\mathrm{Ph} \bullet] \stackrel{\mathrm{PBN}}{\longrightarrow} \mathrm{Ph}_{2} \mathrm{CHN}(\mathrm{O} \bullet) \mathrm{Bu}-t
$$

Decomposition of triphenylbismuth dicrotonate in diffused light in benzene in the presence of spin trap of C-phenyl-N-tert-butylnitrone leads to formation of phenyl radicals which are registered as the adduct $\mathrm{PhCH}(\mathrm{Ph}) \mathrm{N}(0 \bullet) \mathrm{Bu}-t$.

Triphenylbismuth dicrotonate $\mathrm{Ph}_{3} \mathrm{Bi}\left(\mathrm{O}_{2} \mathrm{CCH}=\mathrm{CHCH}_{3}\right)_{2}$ in benzene solution in the presence of $\mathrm{C}$-phenyl-N-tert-butylnitrone $\mathrm{PhCH}=\mathrm{N}(0) \mathrm{Bu}-t$ decomposes in light. An adduct of phenyl radical and spin trap $\mathrm{PhCH}(\mathrm{Ph}) \mathrm{N}\left(\mathrm{O}_{\bullet}\right) \mathrm{Bu}-t$ was registered by ESR method.

Keywords: electron spin resonance; triphenylbismuth dicrotonate; C-phenyl-N-tertbutylnitrone

Received: 01.03.2017; accepted: 03.04.2017; published: 14.07.2017.

А. В. Гущин ${ }^{1}$, О. С. Калистратова ${ }^{1}$, А. И. Малеева ${ }^{1}$, В. А. Куропатов ${ }^{2}$, Д. Н. Емельянов ${ }^{1}$ ${ }^{1}$ Нижегородский государственный университет им. Н. И. Лобачевского, пр. Гагарина, 23, г. Нижний Новгород, 603950, Россия ${ }^{2}$ Институт металлорганической химии им. Г. А. Разуваева РАН, ул. Тропинина, 49, г. Нижний Новгород, 603137, Россия E-mail:gushchin4@yandex.ru

\section{Образование}

\section{mpem-бутилдифенилметилнитроксильного радикала \\ в реакции дикротоната трифенилвисмута и C-фенил-N-mpem-бутилнитрона в бензоле}

$$
\mathrm{Ph}_{3} \mathrm{Bi}(\mathrm{V}) \stackrel{\text { hv }}{\longrightarrow}[\mathrm{Ph} \bullet] \stackrel{\mathrm{PBN}}{\longrightarrow} \mathrm{Ph}_{2} \mathrm{CHN}(\mathrm{O} \bullet) \mathrm{Bu}-t
$$

Распад дикротоната трифенилвисмута на рассеянном свету в бензоле в присутствии спиновой ловушки C-фенил-N-mpem-бутилнитрона приводит к образованию фенильных радикалов, которые регистрируются как аддукт $\mathrm{PhCH}(\mathrm{Ph}) \mathrm{N}(0 \bullet) \mathrm{Bu}-t$. 
Дикротонат трифенилвисмута $\mathrm{Ph}_{3} \mathrm{Bi}\left(\mathrm{O}_{2} \mathrm{CCH}=\mathrm{CHCH}_{3}\right)^{2}$ в присутствии C-фенил-N-mpem-бутилнитрона $\mathrm{PhCH}=\mathrm{N}(0) \mathrm{Bu}-t$ на свету распадается в бензоле. Аддукт фенильного радикала и спиновой ловушки $\mathrm{PhCH}(\mathrm{Ph}) \mathrm{N}(0 \bullet) \mathrm{Bu}-t$ был зафиксирован методом ЭПР.

Ключевые слова: ЭПР; дикротонат трифенилвисмута; C-фенил-N-mpem-бутилнитрон.

Поступило: 01.03.2017; принято: 03.04.2017; опубликовано: 14.07.2017.

(c) Gushchin A. V., Kalistratova 0. S., Maleeva A. I., Kuropatov V. A., Yemelyanov D. N., 2017

\section{Introduction}

Polymethylmethacrylate with organobithmuth compounds additives was known to be obtained with the radical polymerization in the presence of peroxide initiators $[1,2]$. It was established that $\mathrm{Bi}(\mathrm{V})$ acrylates accelerated the polymerization reaction. It was suggested that the acceleration was caused by decomposition of organometallic compounds on exposure by diffused light, as the polymerization slowed in the dark. The character of the process was not studied. When various diacyl derivatives of triphenylbismuth were used, no significant change in polymerization rate or molar mass was observed, indicating the predominant role of the $\mathrm{Ph}_{3} \mathrm{Bi}(\mathrm{V})$ fragment. Besides, the previous study of the photo-induced cation polymerization of oxiranes and vinyl monomers was carried out with triaryl(1-pyrenyl)bismuth salts as initiators $[3,4]$. When such compounds were irradiated by visible light, the homolysis of $\mathrm{Bi}-\mathrm{C}$ (pyrene) bond occured with the formation of pyrenyl radical and cation radical of triarylbismuth, which subsequently initiated polymerization. It is also known that organometallic compounds of bismuth(III) can cause the controlled living radical polymerization $[5,6]$. Recently a phenyl-tert-butylnitroxyl stable radical was fixed in the triphenylbismuth dicro- tonate reaction with 2-methyl-2-nitrosopropane [7]. That result isn't clear proof of the phenyl radical formation by light discharge of organometallic compounds, because of spin trap 2-methyl-2-nitrosopropane in diffused light can be cleavaged to give initial tert-butyl radicals. So, phenyl radical could be a secondary product of homolytic substitution reaction:

$$
\begin{gathered}
t-\mathrm{BuN}=\mathrm{O} \rightarrow t-\mathrm{Bu} \bullet+\mathrm{NO} \\
t-\mathrm{Bu} \bullet+\mathrm{PhBi}(\mathrm{V}) \leftrightarrow t-\mathrm{BuBi}(\mathrm{V})+\mathrm{Ph} \bullet
\end{gathered}
$$

That is why the aim of present work was investigation of triphenylbismuth dicrotonate decomposition reaction in light in the presence of another spin trap C-phenyl-N-tert-butylnitrone which is stable for sun light.

The triphenylbismuth dicrotonate was chosen as organobismuth $(\mathrm{V})$ derivative with good solubility in benzene, hydrolitical and oxidative stability on air. The structure of this compound has been studied [8]. The thermal stability of this compound was investigated by thermogravimetric analysis. The experimental data were used to calculate the standard thermodynamic functions: heat capacity, enthalpy, entropy and Gibbs energy over the range from $T=0$ to $400-420 \mathrm{~K}$. For the compounds under study the standard entropy of formation was calculated at $T=298.15 \mathrm{~K}[9]$. 


\section{Experimental}

ESR spectra were registered on the Bruker ER200D9.5 GHz in degassed tubes.

Benzene, $\mathrm{Et}_{2} \mathrm{O}$ and THF were dried over anhydrous calcium chloride, then distilled and kept over sodium wire. Chloroform was dried over anhydrous calcium chloride and distilled. Hexane was used without previous purification. Anhydrous $\mathrm{BiCl}_{3}$ was purified by sublimation $\left(350^{\circ} \mathrm{C}\right.$, 0.5 torr).

Triphenylbismuth was synthesized according to conventional procedure [10] from $\mathrm{BiCl}_{3}$ and $\mathrm{PhMgBr}$ with the use of the benzene and THF (1:2) mixture as the solvent.

The synthesis of triphenylbismuth dicrotonate was carried out according to conventional procedure [11] by the oxidative addition reaction at room tempera- ture in $\mathrm{Et}_{2} \mathrm{O}$ from triphenylbismuth, crotonic acid and tert-butylhydroperoxide (reagent ratio 1:2:1). The product was purified by recrystallization from the medium hexane - chloroform (4:1). The yield of the purified reaction product was $73 \%$, melting point $153^{\circ} \mathrm{C}$.

The synthesis of PBN performed [12] from benzaldehyde, tert-butylamine and hydrogen peroxide.

Triphenylbismuth dicrotonate decomposition in diffused light was prepared as follows: in one elbow of tube a sample of PBN $(0.2 \mathrm{mmol})$ was placed, while the other elbow was filled by the benzene solution of triphenylbismuth dicrotonate ( $1 \mathrm{~mL}, 0.2 \mathrm{~mol} / \mathrm{L}$ concentration). The ampoule was degassed, sealed and after mixing the ESR spectrum was registered.

\section{Results and Discussion}

We have investigated the decomposition of triphenylbismuth dicrotonate in benzene in the presence of PBN $\mathrm{PhCH}=\mathrm{N}(\mathrm{O}) \mathrm{Bu}-t$ by ESR method in a sealed and degassed ampoule in diffuse light. The choice of the solvent is due to its low activity in radical reactions at the chosen conditions.

We recorded ESR spectrum of the mixture and found an adduct of spin trap $\mathrm{PhCH}(\mathrm{Ph}) \mathrm{N}(\mathrm{O} \bullet) \mathrm{Bu}-t$ with phenyl radi$\mathrm{cal}$, formed by decomposition reaction:

$$
\begin{gathered}
\mathrm{Ph}_{3} \mathrm{Bi}\left(\mathrm{O}_{2} \mathrm{CCH}=\mathrm{CHCH}_{3}\right)_{2} \rightarrow \\
\rightarrow 2 \mathrm{Ph} \bullet+\mathrm{PhBi}\left(\mathrm{O}_{2} \mathrm{CCH}=\mathrm{CHCH}_{3}\right)_{2} \\
\mathrm{Ph} \bullet+\mathrm{PhCH}=\mathrm{N}(\mathrm{O}) \mathrm{Bu}-t \rightarrow \\
\rightarrow \mathrm{PhCH}(\mathrm{Ph}) \mathrm{N}(\mathrm{O} \bullet) \mathrm{Bu}-t
\end{gathered}
$$

The ESR spectrum of triphenylbismuth dicrotonate in benzene (with PBN as the spin trap) is show in Fig. 1.

For the adduct $\mathrm{PhCH}(\mathrm{Ph}) \mathrm{N}(\mathrm{O} \bullet) \mathrm{Bu}-t$ the values of hyperfine interaction constants have been determined: $a_{N}=14.4 \mathrm{Oe}$,
$a_{H}=2.2 \mathrm{Oe}, g=2.0060$, which are in agreement with the literature data for the addition compound generated in benzene by photolysis of $\mathrm{Ph}_{3} \mathrm{Bi}\left(a_{N}=14.5 \mathrm{Oe}, a_{H}=\right.$ $2.3 \mathrm{Oe}), \mathrm{Ph}_{3} \mathrm{Sb}\left(a_{\mathrm{N}}=13.2 \mathrm{Oe}, a_{H}=2.2 \mathrm{Oe}\right)$, $\mathrm{Ph}_{3} \mathrm{As}\left(a_{N}=13.4 \mathrm{Oe}, a_{H}=2.2 \mathrm{Oe}\right)$ [13].

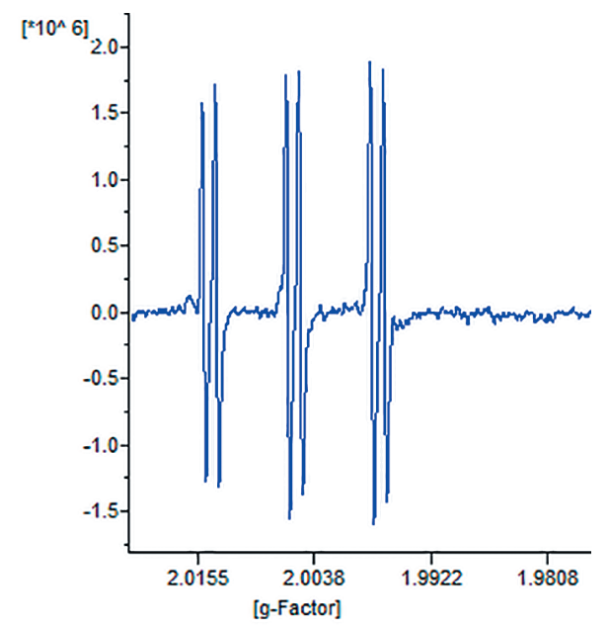

Fig. 1. The ESR spectrum of triphenylbismuth dicrotonate in benzene, the spin trap is $\mathrm{PBN}$ 


\section{Conclusions}

Decomposition of triphenylbismuth dicrotonate in diffused light in benzene solution in the presence of C-phenyl-N- tert-butylnitrone leads to formation of phenyl radicals which are registered in the form of the adduct $\mathrm{PhCH}(\mathrm{Ph}) \mathrm{N}(\mathrm{O} \bullet) \mathrm{Bu}-t$.

\section{Acknowledgements}

This research was supported by Goszadanie RF.

\section{In Russian}

\section{Введение}

Ранее методом радикальной полимеризации в присутствии инициаторов был получен полиметилметакрилат (ПММА) с добавками различных металлоорганических соединений $\mathrm{Bi}(\mathrm{V})[1,2]$. Было установлено, что наличие акрилатов $\mathrm{Bi}(\mathrm{V})$ ускоряет протекание полимеризации. Предположили, что это ускорение вызвано распадом металлоорганических соединений под действием рассеянного света, т. к. в темноте полимеризация замедлялась. При этом характер процесса изучен не был. При использовании различных диацилатных производных трифенилвисмута не наблюдалось значительных изменений в скорости полимеризации и их молекулярной массе, что свидетельствует о превалирующей роли фрагмента $\mathrm{Ph}_{3} \mathrm{Bi}(\mathrm{V})$. Также ранее была изучена фотоиндуцированная катионная полимеризация оксиранов и виниловых мономеров, в которой инициаторами выступали соли триарил(1-пиренил)висмута [3, 4]. При облучении таких соединений видимым светом происходит гомолиз связи Вi-C(пирен) с образованием пиренильного радикала и катион-радикала триарилвисмута, который в дальнейшем и инициирует полимеризацию. Также известно, что металлорганиче- ские соединения висмута(III) способны вызывать контролируемую живую радикальную полимеризацию $[5,6]$. Недавно, фенил-трет-бутилнитроксильный стабильный радикал был зафиксирован в реакции дикротоната трифенилвисмута с 2-метил-2-нитрозопропаном [7]. Этот результат не является четким доказательством образования фенильного радикала при распаде металлоорганического соединения на рассеянном свету, т. к. спиновая ловушка 2-метил-2-нитрозопропан может распадаться с образованием исходных трет-бутильных радикалов. Таким образом, фенильный радикал может быть вторичным продуктом реакции гомолитического замещения:

$$
\begin{gathered}
t-\mathrm{BuN}=\mathrm{O} \rightarrow t-\mathrm{Bu} \bullet+\mathrm{NO} \\
t-\mathrm{Bu} \bullet+\mathrm{PhBi}(\mathrm{V}) \leftrightarrow t-\mathrm{BuBi}(\mathrm{V})+\mathrm{Ph} \bullet
\end{gathered}
$$

Поэтому целью настоящей работы было исследование реакции разложения дикротоната трифенилвисмута на свету в присутствии другой спиновой ловушки C-фенил-N-mpem-бутилнитрона, которая стабильна на солнечном свету.

Дикротонат трифенилвисмута был выбран в качестве металлоорганического производного висмута $(\mathrm{V})$ с хорошей растворимостью в бензоле, гидролитической и окислительной стабильностью 
на воздухе. Структура этого соединения изучена [8]. Термостабильность этого соединения исследовалась с помощью термогравиметрического анализа. Экспериментальные данные были использованы для расчета стандартных термо- динамических функций: теплоемкости, энтальпии, энтропии и энергии Гиббса в диапазоне от $T=0$ до 400-420 К. Для изучаемых соединений стандартная энтропия образования рассчитывалась при $T=298,15$ K [9].

\section{Экспериментальная часть}

ЭПР-спектры регистрировали на приборе Bruker ER200D-SRC с рабочей частотой 9,5 ГГц в дегазированных трубках.

Бензол, $\mathrm{Et}_{2} \mathrm{O}$ и ТГФ сушили над безводным хлоридом кальция, перегоняли и хранили над натриевой проволокой. Хлороформ сушили над безводным хлоридом кальция и перегоняли. Петролейный эфир использовали без предварительной очистки. Безводный $\mathrm{BiCl}_{3}$ очищали сублимацией $\left(350{ }^{\circ} \mathrm{C}\right.$, 0,5 мм рт. ст.).

Трифенилвисмут синтезировали по известной методике [10] из $\mathrm{BiCl}_{3}$ и $\mathrm{PhMgBr}$ с использованием смеси бензола и ТГФ (1:2) в качестве растворителя.

Синтез дикротоната трифенилвисмута проводили по известной методике [11] по реакции окислительного присоединения при комнатной температуре в $\mathrm{Et}_{2} \mathrm{O}$ из трифенилвисмута,

\section{Результаты и обсуждение}

Исследовали распад дикротоната трифенилвисмута методом ЭПР в бензоле в присутствии ФБН $\mathrm{PhCH}=\mathrm{N}(\mathrm{O})$ $\mathrm{Bu}-t$. Выбор растворителя обусловлен тем, что он малоактивен в радикальных реакциях в данных условиях.

Мы зарегистрировали спектр ЭПР смеси и обнаружили аддукт $\mathrm{PhCH}(\mathrm{Ph})$ $\mathrm{N}(\mathrm{O} \bullet) \mathrm{Bu}-t$ спиновой ловушки с фенильным радикалом, образованный в результате реакции разложения: кротоновой кислоты и трет-бутилгидропероксида (соотношение реагентов 1:2:1). Полученный продукт очищали перекристаллизацией из системы: петролейный эфир-хлороформ (4:1). Выход очищенного продукта реакции составил $73 \%$, температура плавления $153^{\circ} \mathrm{C}$.

ФБН синтезировали из бензальдегида, трет-бутиламина и пероксида водорода [12].

Приготовление растворов для исследования распада $\mathrm{Ph}_{3} \mathrm{Bi}\left(\mathrm{O}_{2} \mathrm{CCH}=\mathrm{CHCH}_{3}\right)_{2}$ на рассеянном свету проводили следующим образом. В одно колено Н-образной ампулы для ЭПР помещали навеску ФБН (0,2 ммоль), в другое - раствор $\mathrm{Ph}_{3} \mathrm{Bi}\left(\mathrm{O}_{2} \mathrm{CCH}=\mathrm{CHCH}_{3}\right)_{2} \quad$ в бензоле (1 мл, концентрация 0,2 моль/л). Ампулу дегазировали, запаивали и после смешения содержимого регистрировали спектр ЭПР.

$$
\begin{gathered}
\mathrm{Ph}_{3} \mathrm{Bi}\left(\mathrm{O}_{2} \mathrm{CCH}=\mathrm{CHCH}_{3}\right)_{2} \rightarrow \\
\rightarrow 2 \mathrm{Ph} \bullet+\mathrm{PhBi}\left(\mathrm{O}_{2} \mathrm{CCH}=\mathrm{CHCH}_{3}\right)_{2} \\
\mathrm{Ph} \bullet+\mathrm{PhCH}=\mathrm{N}(\mathrm{O}) \mathrm{Bu}-t \rightarrow \\
\rightarrow \mathrm{PhCH}(\mathrm{Ph}) \mathrm{N}(\mathrm{O} \bullet) \mathrm{Bu}-t
\end{gathered}
$$

ЭПР спектр дикротоната трифенилвисмута в бензоле (спиновая ловушка ФБН) представлен на рис. 1.

Для аддукта $\mathrm{PhCH}(\mathrm{Ph}) \mathrm{N}(\mathrm{O} \bullet) \mathrm{Bu}-t$ определены значения констант сверхтонкого взаимодействия $a_{N}=14,4 Э$, $a_{H}=2,2$ Э, $g=2,0060$, что согласуется 
с литературными данными по этому аддукту, генерируемому в бензоле в ходе фотолиза $\mathrm{Ph}_{3} \mathrm{Bi}\left(a_{N}=14,5\right.$ Э, $\left.a_{H}=2,3 \ni\right), \mathrm{Ph}_{3} \mathrm{Sb}\left(a_{N}=13,2 \ni, a_{H}=2,2 \ni\right)$, $\mathrm{Ph}_{3}$ As $\left(a_{N}=13,4 \ni, a_{H}=2,2\right.$ Э) [13].

\section{Заключение}

Распад дикротоната трифенилвисмута на рассеянном свету в бензоле в присутствии С-фенил-N-mpem-бутилнитрона протекает с образованием фенильных радикалов, которые зафиксированы в форме аддукта $\mathrm{PhCH}(\mathrm{Ph})$ $\mathrm{N}(\mathrm{O} \bullet) \mathrm{Bu}-t$.

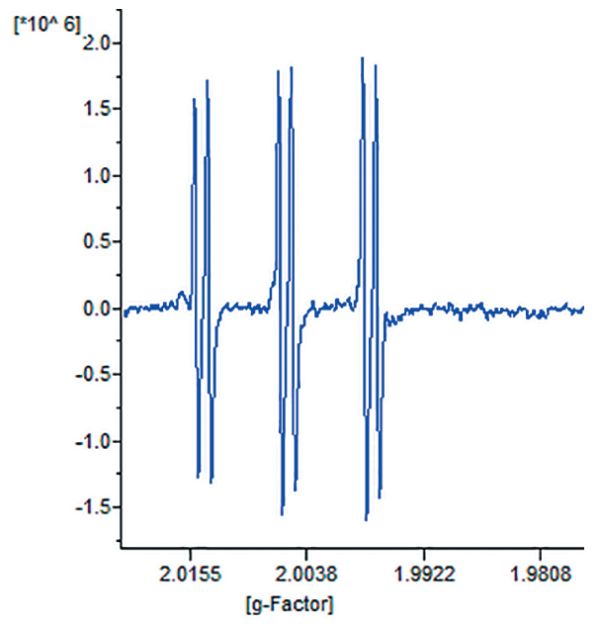

Рис. 1. Спектр ЭПР дикротоната трифенилвисмута в бензоле, спиновая ловушка - ФБН

\section{Благодарности}

Работа выполнена при поддержке Госзадания РФ.

\section{References}

1. Dodonov VA, Gushchin AV, Kuznetsova JL, Morugova VA. [Triphenylbismuth and Triphenylantimony Diacrylates in the Synthesis of Metal-containing Polymethylmethacrylate]. Vestnik Nizhegorodskogo universiteta im. N. I. Lobachevskogo, Seriya Khimiya [Vestnik of Lobachevsky University of Nizhni Novgorod, Ser Chemistry]. 2004;1(4):86-94. Russian.

2. Gushchin AV, Shashkin DV, Shcherbakova TS, Somov NV, Baranov EV, Fukin GK, Shavyrin AS, Rykalin VI, Dodonov VA. [Synthesis and Structure of Tetraphenylantimony Acrylate and its Insertion into Polymethylmethacrylate]. Vestnik Nizhegorodskogo universiteta im. N. I. Lobachevskogo, Seriya Khimiya [Vestnik of Lobachevsky University of Nizhni Novgorod, Ser Chemistry]. 2010;6:68-72. Russian.

3. Matano Y, Shinokura T, Yoshikava O, Imahori H. Triaryl(1-pyrenyl)bismuthonium Salts: Efficient Photoinitiators for Cationic Polymerization of Oxiranes and a Vinyl Ether. Org Lett. 2008;10(11):2167-70. DOI:10.1021/ol8005453.

4. Matano Y. Pentavalent Organobismuth Reagents in Organic Synthesis: Alkylation, Alcohol Oxidation and Cationic Photopolymerization. Top Curr Chem. 2012;311:19-44. DOI:10.1007/128_2011_167.

5. Yamago S. Development of Organotellurium-Mediated and Organostibine-Mediated Living Radical Polymerization Reactions. J Polym Sci, Part A-1: Polym Chem. 2006;44:1-12. DOI:10.1002/pola.21154.

6. Yamago S. Precision Polymer Synthesis by Degenerative Transfer Controlled/Living Radical Polymerization Using Organotellurium, Organostibine, and Organo- 
bismuthine Chain-Transfer Agents. Chem Rev. 200;109(11):5051-68. DOI:10.1021/ cr9001269.

7. Gushchin AV, Kalistratova OS, Maleeva AI, Kuropatov VA. Decomposition of triphenylbismuth dicrotonate in light in the presence of 2-methyl-2-nitrosopropane. Bulletin of the South Ural State University, Series Chemistry. 2016;8(1):51-6. DOI:10.14529/chem 160108 .

8. Andreev PV, Somov NV, Kalistratova OS, Gushchin AV, Chuprunov EV. Bis(but-2enoato- $\kappa O)$ triphenylbismuth(V). Acta Crystallogr Sect E: Crystallogr Commun. 2013;69(6): m333. DOI:10.1107/S1600536813013317.

9. Letyanina IA, Markin AV, Smirnova NN, Klimova MN, Kalistratova OS, Gushchin AV. Calorimetric study of organic compounds of antimony and bismuth $\mathrm{Ph}_{3} \mathrm{Sb}\left(\mathrm{O}_{2} \mathrm{CCH}=\mathrm{CHCH}_{3}\right)_{2}$ and $\mathrm{Ph}_{3} \mathrm{Bi}\left(\mathrm{O}_{2} \mathrm{CCH}=\mathrm{CHCH}_{3}\right)_{2}$. J Therm Anal Calorim. 2016;125(1):339-49. DOI:10.1007/s10973-016-5401-2.

10. Kocheshkov KA, Skoldinov AP, Zemlyanskii NN. Metody elementoorganicheskoy khimii. Sur'ma, vismut [Methods of Organometallic Chemistry. Antimony, Bismuth]. Moscow: Nauka; 1976. 483 p. Russian.

11. Suzuki H. Organobismuth chemistry. Amsterdam-London-New York-Oxford-Paris-Shannon-Tokyo: Elsevier; 2001.619 p.

12. Emmons WD. The Preparation and Properties of Oxaziranes. J Am Chem Soc. 1957;7(5):5739-54. DOI:10.1021/ja01578a043.

13. Xu G, Zhou J, Tang Y. Application of Spin Trapping Technique in Photolysis of Compounds $\mathrm{Ph}_{3} \mathrm{M}(\mathrm{M}=\mathrm{N}, \mathrm{P}, \mathrm{As}, \mathrm{S}$ b, Bi). Wuli Huaxue Xuebao. 1985;1(1):6-11. DOI:10.3866/PKU.WHXB19850102.

\section{Cite this article as (как цитировать эту статью):}

Gushchin AV, Kalistratova OS, Maleeva AI, Kuropatov VA, Yemelyanov DN. Tert-butyl-diphenylmethylnitroxyl radical formation with the reaction of triphenylbismuth dicrotonate and C-phenyl-N-tert-butylnitrone in benzene. Chimica Techno Acta. 2017;4(2):105-111. DOI: 10.15826/chimtech.2017.4.2.025. 